\title{
Spatiotemporal coupling of attosecond pulses
}

\author{
Hampus Wikmark ${ }^{a, 1}$, Chen Guo ${ }^{a, 1}$, Jan Vogelsang ${ }^{a, 1}$, Peter W. Smorenburg ${ }^{b}$, Hélène Coudert-Alteirac ${ }^{a}$, Jan Lahl ${ }^{a}$, \\ Jasper Peschel ${ }^{a}$, Piotr Rudawski ${ }^{a}$, Hugo Dacasa ${ }^{a}$, Stefanos Carlström ${ }^{a, 2}$, Sylvain Maclot ${ }^{a}$, Mette B. Gaarde ${ }^{c}$, \\ Per Johnsson ${ }^{a}$, Cord L. Arnold ${ }^{a}$, and Anne L'Huillier ${ }^{a, 3}$
}

a Department of Physics, Lund University, SE-221 00 Lund, Sweden; b ASML Research, ASML Netherlands B.V., 5504 DR Veldhoven, The Netherlands; and
'Department of Physics and Astronomy, Louisiana State University, Baton Rouge, LA 70803-4001

This contribution is part of the special series of Inaugural Articles by members of the National Academy of Sciences elected in 2018.

Contributed by Anne L'Huillier, January 18, 2019 (sent for review October 12, 2018; reviewed by David Attwood and Margaret M. Murnane)

The shortest light pulses produced to date are of the order of a few tens of attoseconds, with central frequencies in the extreme UV range and bandwidths exceeding tens of electronvolts. They are often produced as a train of pulses separated by half the driving laser period, leading in the frequency domain to a spectrum of high, odd-order harmonics. As light pulses become shorter and more spectrally wide, the widely used approximation consisting of writing the optical waveform as a product of temporal and spatial amplitudes does not apply anymore. Here, we investigate the interplay of temporal and spatial properties of attosecond pulses. We show that the divergence and focus position of the generated harmonics often strongly depend on their frequency, leading to strong chromatic aberrations of the broadband attosecond pulses. Our argument uses a simple analytical model based on Gaussian optics, numerical propagation calculations, and experimental harmonic divergence measurements. This effect needs to be considered for future applications requiring highquality focusing while retaining the broadband/ultrashort characteristics of the radiation.

attosecond pulse | high-order harmonic generation | Gaussian optics | spatiotemporal coupling | focusing of XUV radiation

$E^{2}$ lectromagnetic waves are usually mathematically described by a product of purely spatial and purely temporal terms. This approximation often fails for broadband femtosecond laser pulses (ref. 1 and references therein), and spatiotemporal couplings need to be considered. Spatiotemporal couplings for visible or infrared (IR) light may be introduced by refractive and dispersive elements, such as lenses, gratings, or prisms. The noncollinear amplification in optical parametric crystals may also potentially lead to spatiotemporal couplings, and it is important to develop characterization methods to measure and reduce their effects (2-4). In some cases, these couplings may be advantageously used, as, for example, demonstrated by Vincenti and Quéré (5) for the so-called "lighthouse" effect $(6,7)$.

The shortest light pulses, generated by high-order harmonic generation (HHG) in gases, are in the extreme UV (XUV)/soft $\mathrm{X}$-ray region and in the range of 100 as (8-11), with bandwidths of a few tens or even hundreds of electronvolts (12, 13). These pulses are generated in a three-step process (14, 15). When an atom is exposed to a strong laser field, an electron in the ground state can tunnel through the atomic potential bent by the laser field, propagate in the continuum, and recombine back to the ground state when (and if) returning close to the ionic core. In this process, an XUV photon is emitted, with energy equal to the ionization energy plus the electron kinetic energy at return. Two main families of trajectories leading to the same photon energy can be identified. They are characterized by the "short" or "long" time of travel of the electron in the continuum $(16,17)$. Interferences of attosecond pulses emitted at each laser half-cycle leads to a spectrum of odd-order harmonics.

The investigation of spatiotemporal coupling of attosecond pulses requires measurements of their spatial properties, as a function of time or, equivalently, frequency. Wavefronts of highorder harmonics have been measured by several groups, using different techniques such as Spectral Wavefront Optical Reconstruction by Diffraction (18-20), lateral shearing interferometry (21), point-diffraction interferometry (22), and Hartmann diffraction masks $(23,24)$. In particular, Frumker et al. (25) pointed out that the variation of wavefront and intensity profile with harmonic order leads to spatiotemporal coupling of the attosecond pulses, with temporal properties depending on where they are measured.

The spatial and spectral properties of high-order harmonics strongly depend on the geometry of the interaction and, in particular, on whether the gas medium in which the harmonics are generated is located before or after the focus of the driving laser beam (26). The asymmetry between "before" and "after" can be traced back to the phase of the emitted radiation, which is equal to that of the incident laser field multiplied by the process order, as in any frequency upconversion process, plus the dipole phase which is accumulated during the generation and mostly originates from electron propagation in the continuum. While the former is usually antisymmetric relative to the laser focus, the latter depends on the laser intensity and is therefore symmetric $(21,27)$. The total phase and thus the divergence properties are different before and after the laser focus, leading to a strong dependence of the spatiotemporal properties of the harmonic

\section{Significance}

In most optics textbooks, one writes the electric field describing an optical wave as a product of temporal and spatial amplitudes. This approximation often breaks down for short optical pulses. An example of such spatiotemporal coupling is chromatic aberrations, where the focal properties of the radiation vary with frequency over the pulse bandwidth. In this work, we point out significant chromatic aberrations of attosecond pulses, which depend on the geometry of the generation process. These aberrations are intrinsic to the generation process and need to be eliminated in applications requiring attosecond pulses to be focused over a small region.

Author contributions: H.W., C.G., J.V., P.W.S., H.C.-A., P.R., P.J., C.L.A., and A.L. designed research; H.W., C.G., J.V., P.W.S., H.C.-A., J.L., J.P., P.R., H.D., S.C., S.M., M.B.G., P.J., C.L.A., and A.L. performed research; C.G., J.V., P.W.S., H.C.-A., S.C., S.M., and M.B.G. contributed with analytic tools; H.W., C.G., J.V., P.W.S., H.C.-A., J.P., P.R., S.M., and C.L.A. analyzed data; and H.W. and A.L. wrote the paper.

Reviewers: D.A., University of California, Berkeley; and M.M.M., University of Colorado and NIST.

The authors declare no conflict of interest.

This open access article is distributed under Creative Commons Attribution-NonCommercialNoDerivatives License 4.0 (CC BY-NC-ND).

See QnAs on page 4767

${ }^{1}$ H.W., C.G., and J.V. contributed equally to this work.

${ }^{2}$ Present address: Max Born Institute, 12489 Berlin, Germany.

${ }^{3}$ To whom correspondence should be addressed. Email: anne.Ihuillier@fysik.Ith.se.

Published online March 1, 2019. 
radiation on the generation conditions. In some conditions, harmonics can be emitted with a flat wavefront (21) or even as a converging beam $(28,29)$. Another phenomenon leading to an asymmetry of $\mathrm{HHG}$ with respect to the generation conditions is ionization-induced reshaping of the fundamental field, which depends on whether the beam is converging or diverging when entering the gas medium $(20,30-32)$.

In the present work, we show that the frequency components of attosecond pulses generated by HHG in gases have different divergence properties, which depend on the geometry of the interaction and in particular on where the generating medium is located relative to the laser focus. In some conditions, the position of the focus and divergence strongly vary with frequency, leading to chromatic aberrations, as sketched in Fig. 1, similar to the effect that a chromatic lens has on broadband radiation $(33,34)$. Any imaging optical component will focus the frequency components of the attosecond pulses at different locations, resulting in spatiotemporal couplings. Depending on the position where the pulses are characterized or used, they will have different central frequencies, pulse durations, and spatial widths. We use an analytical expression for the dipole phase (35) combined with traditional Gaussian optics to predict the radius of curvature, position of focus, and divergence of the two trajectory contributions to HHG (29). This model, which assumes generation in a thin slab $(36,37)$, is validated by using numerical simulations of HHG (38) for both thin and thick generating media. We also present experimental measurements of the harmonic divergence as a function of position of generation relative to the laser focus. Finally, we discuss the implications of our results for the focusing of broadband attosecond pulses.

\section{Analytical Expression of the Dipole Phase}

The single-atom response of HHG is well described by an approximate solution of the time-dependent Schrödinger equa-

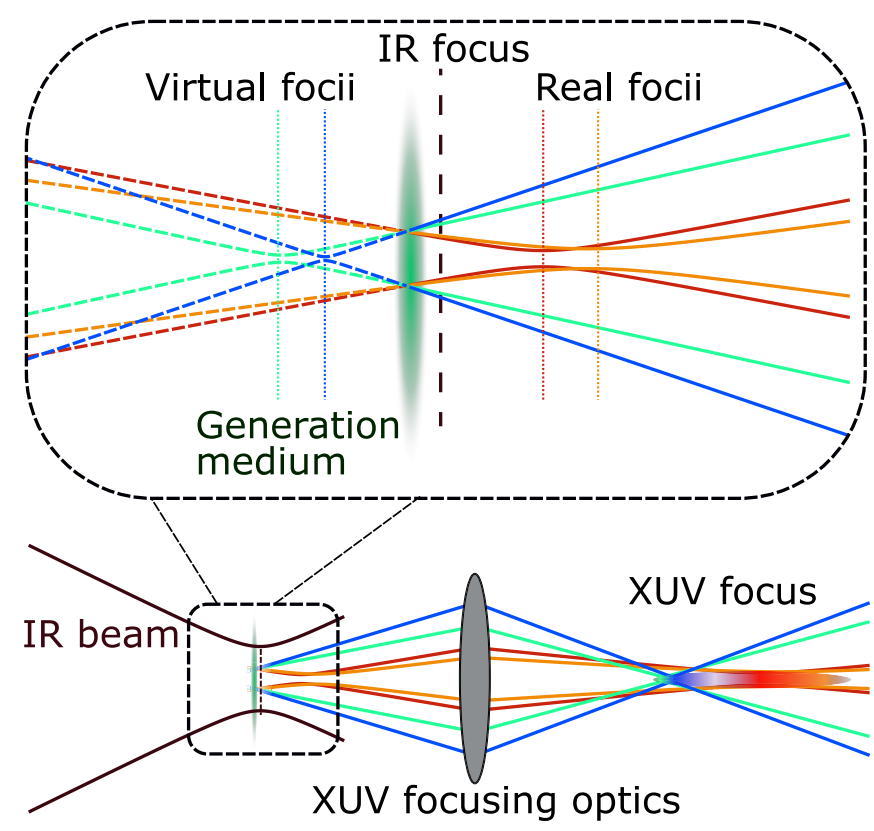

Fig. 1. Illustration of spatiotemporal coupling for an attosecond pulse: different frequencies (harmonic orders 31, 35, 59, and 67, with red, orange, green, and blue colors respectively), generated with varying wavefront curvatures and different divergences, as indicated in Inset, will be refocused by XUV optics (here represented as a lens) at different positions, leading to strong chromatic aberrations and an extended focus, both transversally and longitudinally. The fundamental driving field is indicated by the dark brown/black line. tion (TDSE) for an atom in a strong laser field, called the strong-field approximation (SFA) (39). This theory leads to a simple analytical expression of the dipole phase, equal to $\alpha I$, where $\alpha$ depends on the harmonic order and on the trajectory contributing to $\mathrm{HHG}(16,26,40,41)$ and where $I$ is the laser intensity. This expression has been used in numerous investigations of the harmonic properties $(17,29,41,42)$. Here, we use a more general analytical expression for the phase (35), based on the semiclassical description of attosecond pulse generation $(14,15)$.

In this approximation, the second step of the process is described by solving Newton's equation of motion for a free particle in the laser field. Fig. 2 shows the frequency $(\Omega)$ of the emitted XUV radiation as a function of electron return time for two different fundamental field intensities, indicated by the solid and dashed curves. The frequency varies from $\Omega_{\mathrm{p}}$, corresponding to the ionization threshold $\left(\hbar \Omega_{\mathrm{p}}=I_{\mathrm{p}}, I_{\mathrm{p}}\right.$ denoting the ionization energy and $\hbar$ the reduced Planck constant) to the cutoff frequency $\Omega_{\mathrm{c}}\left(\hbar \Omega_{\mathrm{c}}=3.17 U_{\mathrm{p}}+I_{\mathrm{p}}\right)$. $U_{\mathrm{p}}$ denotes the ponderomotive energy, equal to

$$
U_{\mathrm{p}}=\frac{\alpha_{\mathrm{FS}} \hbar I \lambda^{2}}{2 \pi c^{2} m},
$$

where $\alpha_{\mathrm{FS}}$ is the fine structure constant, $m$ the electron mass, $c$ the speed of light, and $\lambda$ the laser wavelength. The frequency variation can be approximated by piecewise straight lines, as indicated by the black solid lines. After inversion from $\Omega(t)$ to $t(\Omega)$, for each straight line, we have

$$
t_{i}(\Omega)=t_{\mathrm{p} i}+\frac{t_{\mathrm{c} i}-t_{\mathrm{p} i}}{\Omega_{\mathrm{c}}-\Omega_{\mathrm{p}}}\left(\Omega-\Omega_{\mathrm{p}}\right),
$$

where $i=s, \ell$ refers to the electron trajectory (short or long), and $t_{\mathrm{p} i}$ and $t_{\mathrm{c} i}$ are defined as indicated by the dashed black lines in Fig. 2. The values of $t_{\mathrm{p} i}$ and $t_{\mathrm{c} i}$, in both laser cycles and femtoseconds (at $\lambda=800 \mathrm{~nm}$ ), are summarized in Table 1 . We also indicate the return times for the short and long electron trajectories leading to the threshold frequency $\left(t_{\mathrm{ts}}, t_{\mathrm{t} \ell}\right)$ and the return time for the trajectory leading to the cutoff frequency $\left(t_{\mathrm{c}}\right)$. Neglecting the frequency dependence of the time for tunneling and recombination, $t_{i}(\Omega)$ can be interpreted as the group delay of the emitted radiation. Its integral is the spectral phase

$$
\Phi_{i}(\Omega)=\Phi_{i}\left(\Omega_{\mathrm{p}}\right)+t_{\mathrm{p} i}\left(\Omega-\Omega_{\mathrm{p}}\right)+\frac{t_{\mathrm{c} i}-t_{\mathrm{p} i}}{\Omega_{\mathrm{c}}-\Omega_{\mathrm{p}}} \frac{\left(\Omega-\Omega_{\mathrm{p}}\right)^{2}}{2} .
$$

As shown in Fig. 2, the return times $t_{\mathrm{p} i}, t_{\mathrm{c} i}$, and therefore the second term in Eq. 3 do not depend on laser intensity. Using $\Omega_{\mathrm{c}}-\Omega_{\mathrm{p}}=3.17 U_{\mathrm{p}} / \hbar$, the coefficient in the third term can be written as

$$
\frac{t_{\mathrm{c}}-t_{\mathrm{p} i}}{\Omega_{\mathrm{c}}-\Omega_{\mathrm{p}}}=\frac{2 \gamma_{i}}{I}
$$

where

$$
\gamma_{i}=\frac{\left(t_{\mathrm{c} i}-t_{\mathrm{p} i}\right) \pi c^{2} m}{3.17 \alpha_{\mathrm{FS}} \lambda^{2}} .
$$

In this classical calculation, $\Phi_{i}\left(\Omega_{\mathrm{p}}\right)$ is equal to zero for the short trajectory, while it is proportional to the laser intensity for the long: $\Phi_{\ell}\left(\Omega_{\mathrm{p}}\right)=\alpha_{\ell} I$. The value of $\alpha_{\ell}$ can be obtained numerically within the classical approach used in this work (43) and is found to be close to that given within the SFA, equal to $4 \pi^{2} \alpha_{\mathrm{FS}} / m \omega^{3}$, where $\Omega$ is the laser frequency (16, 41). The parameters needed to describe $\Phi_{i}(\Omega)$ for $800-\mathrm{nm}$ radiation are $\gamma_{\mathrm{s}}=1.03 \times 10^{-18} \mathrm{~s}^{2} \cdot \mathrm{W} \cdot \mathrm{cm}^{-2}, \gamma_{\ell}=-0.874 \times$

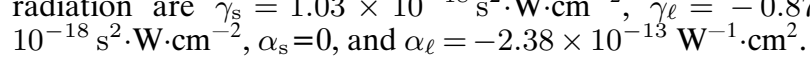




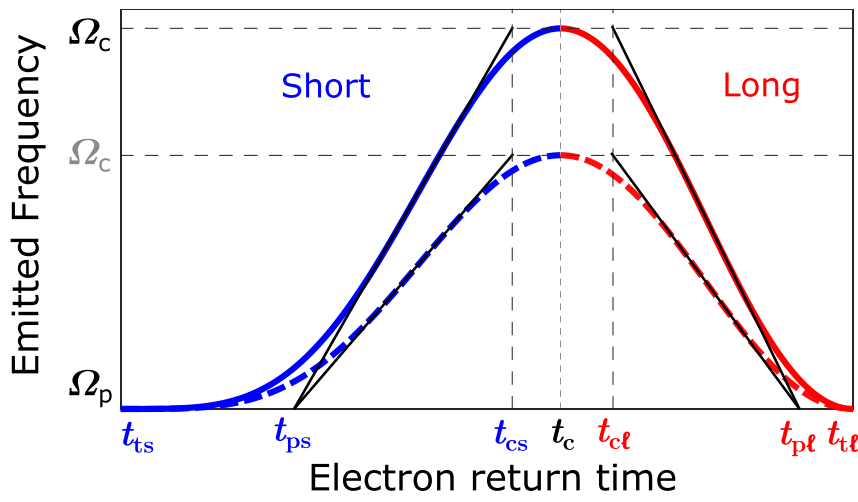

Fig. 2. Emitted XUV frequency as a function of return time for two laser intensities, corresponding to the solid and dashed blue/red curves. The blue curves describes the short trajectories, while the red lines refer to the long trajectory. $t_{t s, t \ell}$ are the return times for the short and long electron trajectories leading to the threshold frequency $\Omega_{\mathrm{p}}$. $t_{\mathrm{c}}$ is the return time for the trajectory leading to the cutoff frequency $\Omega_{\mathrm{c}} . t_{\mathrm{p} i}$ and $t_{\mathrm{c} i}(i=\mathrm{s}, \ell)$ are return times obtained by approximating $\Omega(t)$ as piecewise straight lines. Values for these return times are indicated in Table 1.

The dipole phase can be approximated for the two families of trajectories by the expansion:

$$
\Phi_{i}(\Omega)=\alpha_{i} I+t_{\mathrm{p} i}\left(\Omega-\Omega_{\mathrm{p}}\right)+\frac{\gamma_{i}}{I}\left(\Omega-\Omega_{\mathrm{p}}\right)^{2} .
$$

The present expression gives very similar results to, e.g., the numerical results presented in ref. 40, obtained by solving saddlepoint equations within the SFA, with the advantage of being analytical.

\section{Wavefront and Spatial Width of XUV Radiation}

We now use this analytical expression for the dipole phase together with traditional Gaussian optics to predict the radius of curvature, position of focus, and divergence of the two trajectory contributions to HHG. A similar derivation has been proposed, independently, by Quintard et al. (29) with, however, a different analytical formulation of the dipole phase. We neglect the influence of propagation, considering an infinitely thin homogeneous gas medium $(36,37,44)$. Such an approximation is valid in a loose focusing geometry, where the generating medium length is much smaller than the Rayleigh length. We also assume that the fundamental field is Gaussian, with intensity $I(r, z)$, radial width $w(z)$ at $1 / e^{2}$, radius of curvature $R(z)$, and peak intensity $I_{0}, z$ denoting the coordinate along the propagation axis and $r$ the radial coordinate. The focus position is $z=0$ and the waist $w_{0}=w(0)$. Considering only the contribution of one trajectory $i$, the phase of the $q$ th harmonic field can be approximated by

$$
\Phi_{q}(r, z)=q \phi(r, z)+\Phi_{i}(r, z) .
$$

The phase of the fundamental Gaussian beam is $\phi(r, z)=k z-$ $\zeta(z)+k r^{2} / 2 R(z)$, where $k$ is the wavevector equal to $\omega / c$ and $\zeta(z)$ the Gouy phase (45). This article is mainly concerned with the third term, giving the curvature of the beam. The dipole phase $\Phi_{i}(r, z)$ is given by Eq. 6, for $I=I(r, z)$ and $\Omega=q \omega$. Omitting the second term in Eq. 6, which does not depend on intensity and therefore on space, $\Phi_{i}(r, z)$ can be expressed as

$$
\Phi_{i}(r, z)=\frac{\alpha_{i} I_{0} w_{0}^{2}}{w^{2}(z)} e^{-\frac{2 r^{2}}{w^{2}(z)}}+\frac{\gamma_{i}\left(\Omega-\Omega_{p}\right)^{2} w^{2}(z)}{I_{0} w_{0}^{2}} e^{\frac{2 r^{2}}{w^{2}(z)}} .
$$

We use a Taylor expansion close to the center of the beam to approximate $\Phi_{i}(r, z)$ (Eq. 8). To determine the harmonic wavefront, we only keep the terms proportional to $r^{2}$ in Eq. $\mathbf{8}$, to which we add the $r^{2}$-dependent contribution from the fundamental, equal to $q k r^{2} / 2 R(z)$. The resulting $r^{2}$-dependent contribution to the phase of the harmonic field can be written as $q k r^{2} / 2 R_{i}$, with

$$
\frac{1}{R_{i}}=\frac{1}{R(z)}-\frac{4 \alpha_{i} I_{0} w_{0}^{2} c}{w^{4}(z) \Omega}+\frac{4 \gamma_{i}\left(\Omega-\Omega_{p}\right)^{2} c}{I_{0} w_{0}^{2} \Omega} .
$$

For simplicity of the notations, we omit to explicitly indicate the $z$ dependence of $R_{i}$. The curvature of the harmonic field is equal to that of the fundamental (first term) plus that induced by the dipole phase. The second term is only present for the long trajectory. This equation outlines the dependence of the XUV radiation wavefront on frequency $(\Omega)$, electron trajectory $(i)$, intensity at focus $\left(I_{0}\right)$, and generation position $(z)$. Eq. 9 is illustrated in Fig. $3 A$, representing the wavefronts induced by the fundamental (black) and due to the dipole phase for the short trajectory (green) as a function of the generation position. The fundamental wavefront changes from convergent to divergent through the focus, while that induced by the dipole phase is always divergent and independent of the generation position $(z)$.

Using the reduced coordinate $Z=z / z_{0}$, where $z_{0}=\pi w_{0}^{2} / \lambda$ is the fundamental Rayleigh length, Eq. 9 can be written as

$$
\frac{z_{0}}{R_{i}}=\frac{1}{Z+1 / Z}-\frac{\eta_{i}}{\left(1+Z^{2}\right)^{2}}+\mu_{i},
$$

where $\eta_{i}=2 \alpha_{i} I_{0} / q$ and $\mu_{i}=2 \gamma_{i} \omega^{2}\left(q-q_{\mathrm{p}}\right)^{2} / q I_{0}$ are dimensionless quantities $\left(q_{\mathrm{p}}=\Omega_{\mathrm{p}} / \omega\right)$. For the short trajectory, since $\alpha_{\mathrm{s}}=$ 0 , the positions where the radius of curvature diverges, corresponding to a flat phase front, can be calculated analytically by solving a second-order equation in $Z$,

$$
Z^{2}+\frac{Z}{\mu_{\mathrm{s}}}+1=0
$$

For $\mu_{\mathrm{s}} \leq 0.5$, the solutions to this equation are real and the radius of curvature diverges at

$$
Z_{ \pm}=-\frac{1}{2 \mu_{\mathrm{s}}} \pm \sqrt{\frac{1}{4 \mu_{\mathrm{s}}^{2}}-1}
$$

This discussion is illustrated graphically in Fig. $3 B$ for the $23 \mathrm{rd}$ harmonic of 800-nm radiation generated in Ar, with $I_{0}=3 \times$ $10^{14} \mathrm{~W} \cdot \mathrm{cm}^{-2}$. In these conditions, we have $\eta_{\mathrm{s}}=0, \mu_{\mathrm{s}}=0.253$, $\eta_{\ell}=-6.38$, and $\mu_{\ell}=-0.215$. Fig. $3 B$ presents the radius of curvature in reduced units $R_{i} / z_{0}$ for the short (blue) and long (red) trajectory contributions. Over the range shown in the

Table 1. Return times for the short and long trajectories relative to the zero of the electric field

\begin{tabular}{llcc} 
Return time & \multicolumn{1}{c}{ Brief description } & Cycle & fs \\
\hline$t_{\mathrm{ts}}$ & Short, threshold & 0 & 0 \\
$t_{\mathrm{ps}}$ & Short, threshold, model & 0.18 & 0.48 \\
$t_{\mathrm{cs}}$ & Short, cut-off, model & 0.40 & 1.07 \\
$t_{\mathrm{c}}$ & Cut-off & 0.45 & 1.20 \\
$t_{\mathrm{c} \ell}$ & Long, cut-off, model & 0.50 & 1.35 \\
$t_{\mathrm{p} \ell}$ & Long, threshold, model & 0.69 & 1.85 \\
$t_{\mathrm{t} \ell}$ & Long, threshold & 0.75 & 2.00 \\
\hline
\end{tabular}

For the last column, a laser wavelength of $800 \mathrm{~nm}$ is used. 
figure, between $-2 z_{0}$ and $z_{0}, R_{\mathrm{s}} / z_{0}$, represented by the blue curve, diverges at $Z_{+}=-0.272$. The other solution of Eq. 11 is $Z_{-}=-3.68$ which is outside the scale of the figure. For the long trajectory, the radius of curvature, represented by the red solid line, diverges at $Z \simeq-1.4$. This behavior is quite general for all harmonics, as discussed in the last section of this work.

To estimate in a simple way the spatial width of the harmonic field at the generation position, we assume that its amplitude is proportional to the fundamental amplitude to a power $p(36,37$, $44,46,47)$. This exponent is quite constant in the plateau region and typically of the order of 4 , as confirmed by our TDSE calculations presented below. The harmonic width is then simply equal to $W=w(z) / \sqrt{p}$ (here, as well, we omit to write explicitly the $z$-dependence of $W$ ).

\section{Focus Position and Beam Waist}

Knowing the beam radius of curvature and width at a given position $z$, it is a simple exercise within Gaussian optics to determine the position of the focus and the corresponding waist (e.g., ref. 45 ). The position of focus relative to the generation position $z$ is given by

$$
z_{i}=-\frac{R_{i}}{1+\left(\lambda_{q} R_{i} / \pi W^{2}\right)^{2}},
$$

with $\lambda_{q}=\lambda / q$. By using reduced coordinates relative to the fundamental Rayleigh length, Eq. 13 can be written as

$$
\frac{z_{i}}{z_{0}}=-\frac{R_{i}}{z_{0}}\left(1+\left[\frac{p R_{i}}{q z_{0}\left(1+Z^{2}\right)}\right]^{2}\right)^{-1}
$$

The corresponding waist at focus is

$$
w_{i}=\frac{W}{\sqrt{1+\left(\pi W^{2} / \lambda_{q} R_{i}\right)^{2}}}
$$

or, relative to the fundamental waist,

$$
\frac{w_{i}}{w_{0}}=\left(\frac{1+Z^{2}}{p}\right)^{\frac{1}{2}}\left(1+\left[\frac{q z_{0}\left(1+Z^{2}\right)}{p R_{i}}\right]^{2}\right)^{-\frac{1}{2}}
$$

Fig. 4 shows the position of the harmonic focus $\left(z_{i} / z_{0}\right)$ relative to that of the generation position $\left(z / z_{0}\right)(A)$ and the normalized far-field divergence $\theta_{i} / \theta_{0}=w_{0} / w_{i}(B)$ for the two trajectories, short (blue solid line) and long (red solid line). The color plots represent harmonic intensities obtained from a simulation presented in Numerical Calculations. The divergence of the fundamental $\theta_{0}$ is defined as $\lambda / \pi w_{0}$. Let us emphasize that the zero of the horizontal scale is the laser focus, while in $A$, zero on the vertical scale means that the focus of the harmonic field coincides with the generation position. The focus position and divergence strongly vary with $z$ and quite differently for the two trajectories. In both cases, the focus position changes sign, and the divergence goes through a minimum when the radius of curvature goes to infinity (Fig. 3).

For the short trajectory and $Z \leq Z_{+}$, the focus is real, and it is located after the generation position $\left(z_{i} \geq 0\right)$ along the propagation direction. The negative curvature of the convergent fundamental beam is larger in magnitude than the positive curvature induced by the dipole phase, and the harmonics are generated as a convergent beam (29). When $Z>Z_{+}$, the focus is virtual and located before the generation position. Two cases can be considered: When $0>Z>Z_{+}$, i.e., when the generation position is before the IR focus, the negative curvature of the fundamental beam is smaller in magnitude than the positive curvature induced by the dipole phase: The harmonics are generated as a divergent beam. When $Z \geq 0$, both curvatures are positive, and the harmonics are generated as a divergent beam. The divergence is smallest in the region close to $Z_{+}$.

The same reasoning applies for the long trajectory contribution, except that $Z_{+}$is now replaced by $Z \approx-1.4$ (Fig. 3 ). In this case, in the region with enough intensity for HHG, i.e., $|Z| \leq 1.5$, corresponding to $I=9 \times 10^{13} \mathrm{~W} \cdot \mathrm{cm}^{-2}$, the harmonic focus is located just before the generation position, and the divergence is much larger than that of the short trajectory contribution.

At the positions where the radius of curvature diverges (indicated by the dashed line in Fig. 4 for the short trajectory), the harmonics are generated with a flat wavefront and with a large focus (low divergence). In contrast, harmonics generated far away from the divergence minima will inherit a curvature from the fundamental and the dipole phase contribution which corresponds to a significantly smaller beam waist in the real or virtual focus and thus in a significantly larger divergence. The variation of the divergence with generation position is due partly to the dipole phase contribution, but also to the mismatch between the harmonic order $q$ and the amplitude variation here described by a power law with exponent $p=4$ (Eq. 16).

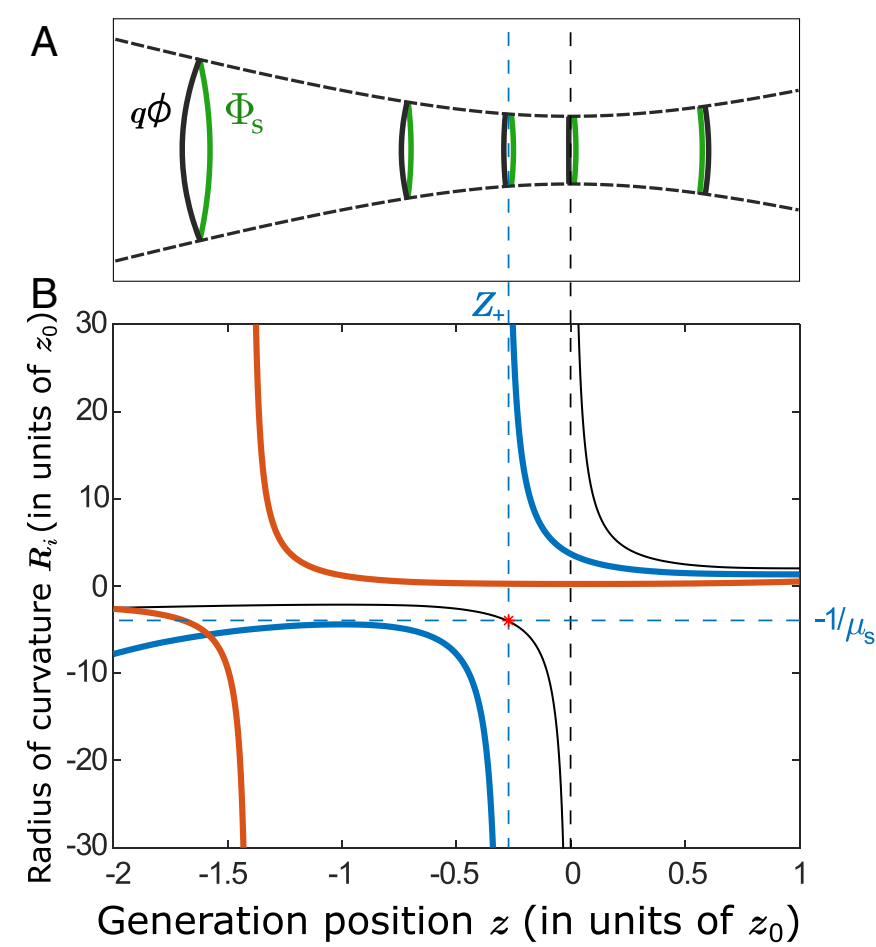

Fig. 3. (A) Representation of different contributions to the harmonic wavefront, due to the fundamental (black) and due to the dipole phase for the short trajectory (green) at different generation positions ( $z$ ). The fundamental beam profile variation is indicated by the thick black dashed line. (B) Radius of curvature of the 23rd harmonic as a function of generation position. The laser wavelength is $800 \mathrm{~nm}$, and the peak intensity at focus is $3 \times 10^{14} \mathrm{~W} \cdot \mathrm{cm}^{-2}$. The blue (red) solid line is obtained for the short (long) trajectory. The thin solid line shows the radius of curvature of the fundamental. At the position $Z_{+}$, where $R(z)=-z_{0} / \mu_{\mathrm{s}}, R_{\mathrm{s}} / z_{0}$ diverges. As can be seen in $A$, this is when the two phase contributions cancel out, as shown by the horizontal blue dashed line. In both $A$ and $B$, the vertical thin dashed lines indicate the position of the harmonic focus (for the short trajectory, in blue) and the fundamental focus (black). The symbols are defined in the text; Eqs. 7, 10, and 12. 


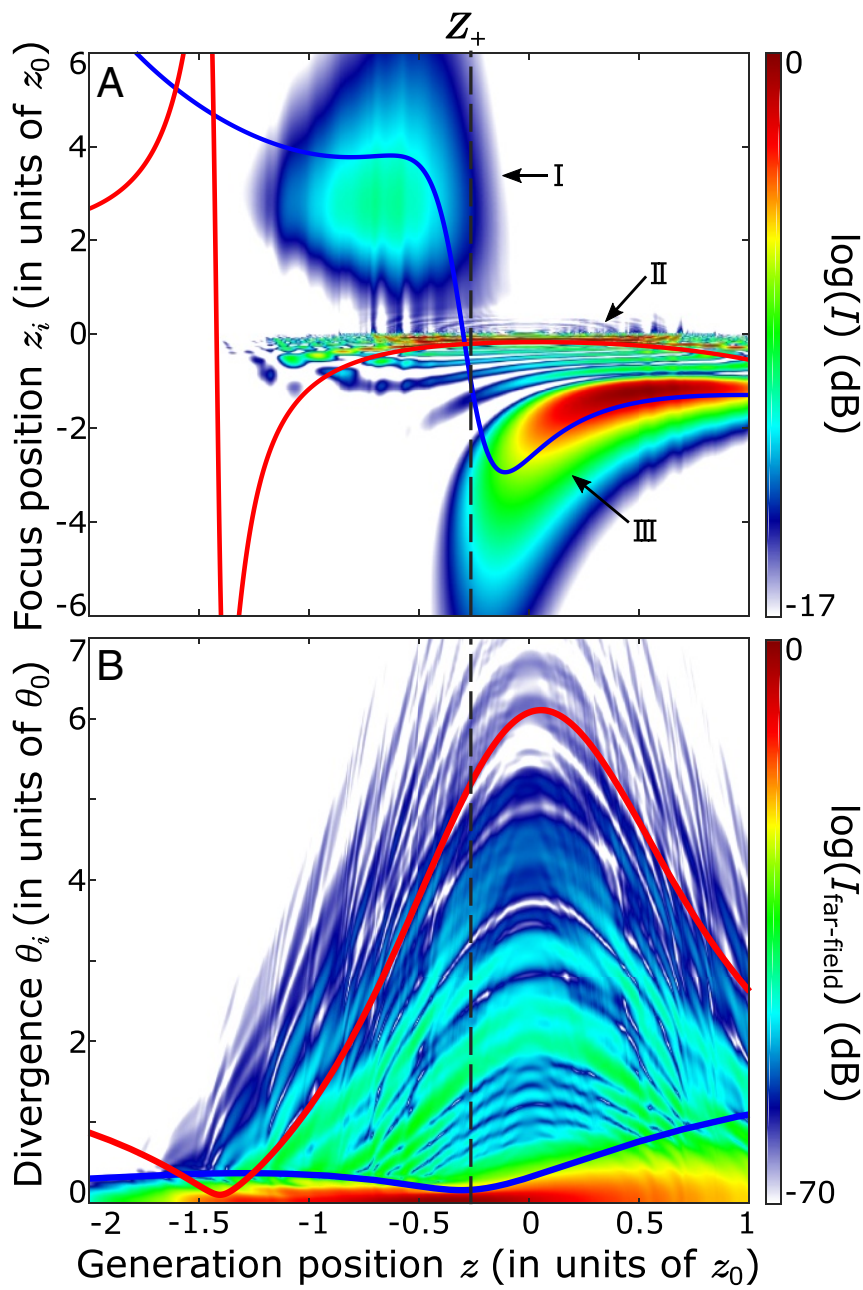

Fig. 4. Position of the focus of the 23 rd harmonic relative to the generation position $(A)$ and far-field divergence $(B)$ as a function of the generation position relative to the laser focus. The results for the short and long trajectory are indicated by the blue and red curves, respectively. The dashed line corresponds to the position $Z_{+}$, where the radius of curvature for the short trajectory diverges. The color plots indicate results of a calculation based on the solution of the TDSE, where HHG is assumed to occur in an infinitely thin plane. In $A$, the on-axis intensity at a certain position along the propagation axis is plotted as a function of generation position on a logarithmic scale. Three different focal regions, labeled I-III can be identified. In $B$, the radial intensity calculated at a distance of $50 z_{0}$ from the generation position, long enough to reach the far field, and normalized to the fundamental radial intensity at the same distance is indicated.

\section{Numerical Calculations}

To validate the Gaussian model presented in this work, we performed calculations based on single-atom data obtained by solving the TDSE for a single active electron in Ar exposed to a constant intensity. The time-dependent dipole response was calculated for 5,000 intensity points. This allows us, for each harmonic frequency, to precisely unwrap the amplitude and phase variation as a function of intensity, and thus to accurately describe the interferences of the trajectories. The complex electric-field distribution at a given harmonic frequency is obtained by integrating in time the polarization induced by the fundamental field in an arbitrarily thin sheet of homogeneous Ar gas. The field is then propagated to different positions relative to the generation position by calculating the diffraction integral in Fresnel approximation using Hankel transforms. The influence of ionization is not taken into account. This procedure is repeated for different gas target positions rel- ative to the laser focus. We use a fundamental wavelength of $800 \mathrm{~nm}$, a pulse duration of $45 \mathrm{fs}$, and a peak intensity of $3 \times 10^{14} \mathrm{~W} \cdot \mathrm{cm}^{-2}$.

Fig. $4 A$ presents a color plot of the 23 rd harmonic on-axis intensity for different generation positions (horizontal axis). The regions with the warmest colors (i.e., toward red) represent the focal regions. The small regions with high peak intensity (dark red, like that labeled II) correspond to the smallest focus. The agreement between the numerical predictions and those of the Gaussian model is striking. When $Z \leq Z_{+}$, the 23rd harmonic is focused after the generation position (region I). When $Z \geq Z_{+}$, two focal regions can be identified, a very thin one close to the generation position (region II) and a larger one at larger negative $z_{i}$ (region III). The agreement with the results of the Gaussian model allows us to interpret the main contribution to these regions: short trajectory for I and III and long trajectory for II. While the focus position for the long trajectory contribution remains close to (just before) the generation plane, the focus position of the short trajectory contribution strongly depends on the generation position. The harmonic radiation often exhibits two foci, due to the two trajectories.

Fig. $4 A$ presents a series of interference structures, some vertical, others almost horizontal. To identify the physical reason for these structures, we have performed simulations allowing us to separate the contributions of the trajectories, using the thin medium approximation and harmonic fields as in our model. Instead of Gaussian optics, however, we used diffraction integrals for the propagation. These simulations show that the horizontal fringes (e.g., between regions II and III) concern the short trajectory contribution and come from the fact that the harmonic phase front and intensity profile are not those of a Gaussian beam (37). The vertical features (e.g., between I and II), however, are a manifestation of quantum path interferences $(41,42)$, since they only appear when both contributions are coherently added.

The color plot in Fig. $4 B$ is the 23rd harmonic radial intensity at a distance of $50 z_{0}$, as a function of generation position. This distance is long enough to reach the far field region, so that the radial intensity is proportional to the far field divergence. As for the focus position, the comparison with the prediction of the Gaussian model allows us to distinguish the contribution of the two trajectories, with quite different divergence, especially for $|Z| \leq 1$. The red (blue) curves represent the $1 / e^{2}$ divergence within the Gaussian model for the long (short) trajectories. The blue-green colored regions in $B$ can be attributed to the long trajectory, while the red-yellow-bright green regions are due to the short trajectory.

An important question is whether these results are still valid after propagation in a finite medium. We used the single-atom data described as input in a propagation code based on the slowly varying envelope and paraxial approximations (38). We present in Fig. 5 results obtained for a 5.4-mm-long $(A)$, 30mm-long $(B)$, and 60-mm-long $(C)$ homogeneous medium, using a 2-mbar gas pressure and a fundamental waist size of $w_{0}=$ $350 \mu \mathrm{m}$. While Fig. $5 A$ compares very well with the results shown in Fig. $4 A$, as expected, Fig. $5 B$ and $C$ shows clear effects of propagation, related to ionization-induced defocusing of the fundamental laser beam. In fact, two different phase-matching regimes appear: one similar to what is present in absence of propagation and which agrees well with the predictions of the Gaussian model (compare regions I and III in Fig. $5 A$ and $B$ ), and a second one, which also follows a similar model but for a fundamental focus moved to the left (see regions I' $^{\prime}$ and III' $^{\prime}$ in Fig. $5 B$ ), as expected for a fundamental beam that is defocused due to partial ionization of the medium (20, 30-32). To examine in more details the effect of propagation goes beyond the scope of this paper. 
$A$

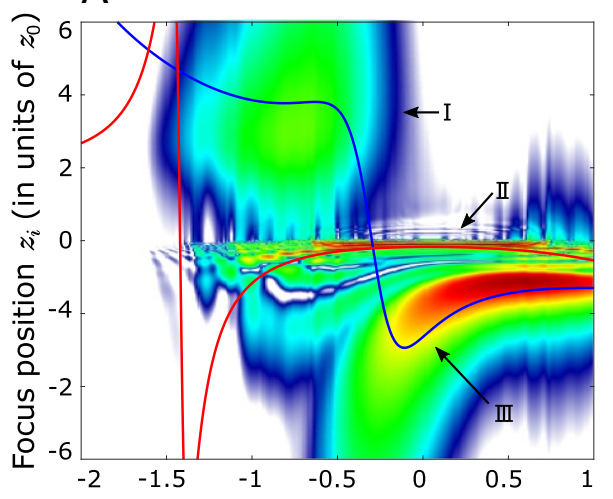

B

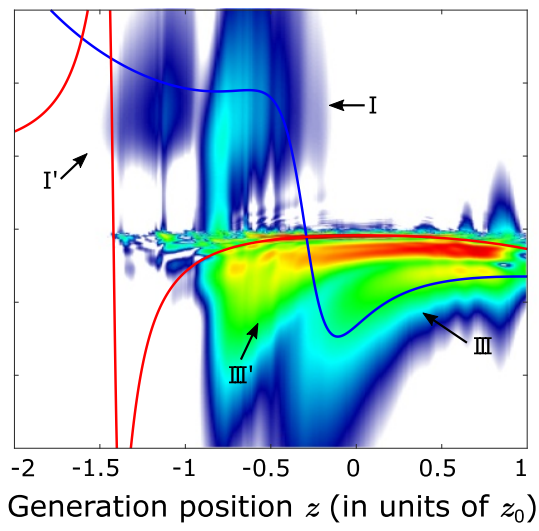

C

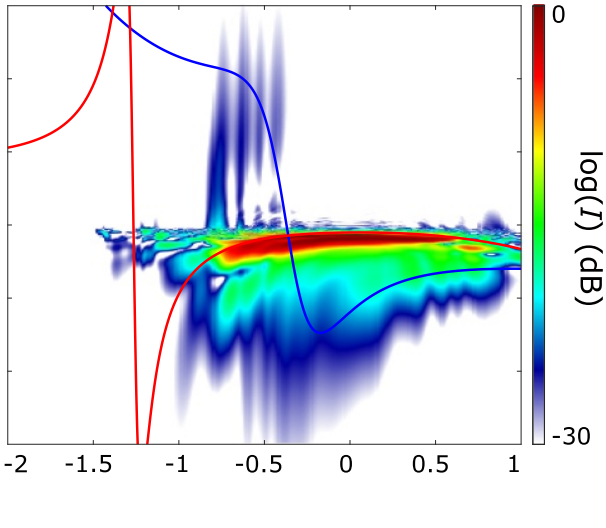

Fig. 5. Results of propagation calculations for the 23rd harmonic for a 5.4-mm-long $(A)$, 30-mm-long $(B)$, and 60-mm-long (C) gas cell. The on-axis intensity at a certain position along the propagation axis is plotted as a function of generation position on a logarithmic scale. The results of the Gaussian model are indicated by the blue and red solid lines for the short and long trajectories and are identical to those of Fig. $4 \mathrm{~A}$.

\section{Experimental Divergence Measurements}

Experiments were performed at the intense XUV beamline of the Lund Laser Centre $(48,49)$, by using a multiterawatt 45 -fs titanium-sapphire laser operating at a $10-\mathrm{Hz}$ repetition rate. The beam was (slightly) apertured to $27 \mathrm{~mm}$ and focused by using a spherical mirror with focal length $f=8 \mathrm{~m}$. The laser aberrations were minimized by using a deformable mirror coupled to an IR Shack-Hartmann wavefront sensor. The harmonics were generated in a $60-\mathrm{mm}$ gas cell filled with Ar by a pulsed valve. We measured the divergence of the emitted harmonics using a flat-field XUV spectrometer with an entrance slit located approximately $6 \mathrm{~m}$ after the generation. For each harmonic, the width was estimated by fitting a Gaussian function onto the transverse (spatial) direction of the spectrometer. The IR focus was moved relative to the gas cell along the direction of propagation by changing the voltage of the actuator which controls the curvature of the deformable mirror. The limits of the scan were imposed by the decrease of the harmonic yield, which is slightly asymmetric relative to the laser focus (26).

The widths of the 13th-19th harmonics are shown in Fig. $6 A$ and compared with theoretical predictions in $B-D$, obtained by using a laser waist of $220 \mu \mathrm{m}$ and a maximum intensity of $2.5 \times$ $10^{14} \mathrm{~W} \cdot \mathrm{cm}^{-2}$ (the Rayleigh length is estimated to $0.2 \mathrm{~m}$ ). The harmonic widths were calculated as $\left(z_{i}+L\right) \theta_{i}$, where $L=6 \mathrm{~m}$ is the distance from the gas cell to the measurement point. Fig. $6 B$ presents results of numerical calculations based on solving the TDSE and the propagation equations, using parameters mimicking the experimental conditions as well as possible. In Fig. $6 C$, the results of the Gaussian model for the short trajectory are shown, while in Fig. $6 D$, a "truncated" Gaussian model is used, where the expressions for the beam waist, radius of curvature, and intensity variation of the fundamental beam now include the effect of a circular aperture $(50,51)$, taken to be equal to the experimental one. Going from the left to the right in all of the plots in Fig. 6, the harmonic widths first decrease (or stay approximately constant for the highest orders) and then increase. The harmonic widths vary more strongly in the Gaussian model than in the other calculations and in the experiment. We investigated the reason for this difference by varying the parameters used in the propagation simulations, such as medium length, gas pressure, aperture diameter, and pulse energy. Unlike the conditions used in Fig. $5 B$ and $C$, effects due to propagation, e.g., induced by ionization-defocusing, are negligible, and the main reason for the difference between Fig. $6 B$ and $C$ is the beam truncation due to the aperture, as confirmed by Fig. $6 D$. Effects due to propa- gation in the nonlinear medium, which become nonnegligible at higher laser intensity, actually lead to faster variation of the beam divergence on both sides of the laser focus.

\section{Chromatic Aberrations of Attosecond Pulses}

Finally, we study the variation of the focus position and beam waist over a large spectral bandwidth. To obtain a broad spectral region, we consider generation of high-order harmonics in neon atoms. HHG spectra obtained in $\mathrm{Ne}$ (52) are broader and flatter than those in Ar, which exhibit a strong modulation due to a Cooper minimum at $\sim 45 \mathrm{eV}$. Fig. 7 shows the predictions of the Gaussian model, for the 31st to the 71st harmonics of $800-\mathrm{nm}$ radiation, at an intensity of $5 \times 10^{14} \mathrm{~W} \cdot \mathrm{cm}^{-2}$. We only consider here the contribution from the short trajectory. The Gaussian model is used here for simplicity. It should be

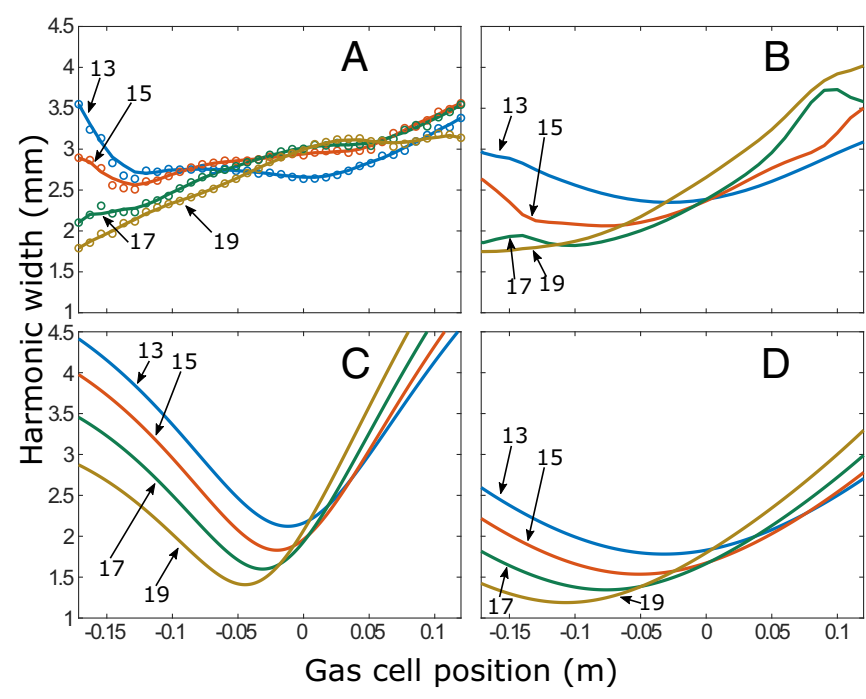

Fig. 6. (A) Spatial widths of harmonics 13-19 generated in Ar and measured approximately $6 \mathrm{~m}$ after generation as a function of the cell position. The solid lines are fit to the experimental data indicated by the circles. $(B-D)$ Spatial widths of the same harmonics as a function of generation position, obtained by the numerical simulations solving the TDSE and the propagation equations $(B)$ and predicted by the Gaussian model for the short trajectory $(C)$ and the same model using a truncated Gaussian beam $(D)$ The peak intensity in vacuum is $2.5 \times 10^{14} \mathrm{~W} \cdot \mathrm{cm}^{-2}$, and the laser beam waist is $220 \mu \mathrm{m}$. 
reasonably accurate in conditions of thin medium, not too high degree of ionization, which is the case for $\mathrm{Ne}$ atoms at the intensity used, and high spatial quality, nontruncated, fundamental beam.

The variation of the focus position as a function of generation position strongly depends on the process order. This is due to the frequency dependence of Eq. 9 and, in particular, depends on whether the radius of curvature diverges. Since $\mu_{\mathrm{s}}$ increases with frequency, the two zeros $Z_{ \pm}$of Eq. 9 move closer to each other, as is clear in Fig. $7 A$ by comparing, e.g., harmonics 41 and 43 ( $Z_{ \pm}$correspond to the two maxima in the figure). At a certain frequency, corresponding to harmonic 45 in Fig. $7 A,-1 / \mu_{\mathrm{s}}$ becomes tangent to $R(z)$ at $z=-z_{0}$ (see also Fig. 3). Above this frequency, the radius of curvature does not diverge and remains negative. The harmonic focus position is then always located before the generation position. As $-1 / \mu_{\mathrm{s}} \rightarrow$ 0 , when the frequency increases, the focus position becomes largely independent from the generation. In this region, the harmonics are much more focused, as shown by the blue lines in Fig. $7 B$.

To estimate the consequence of these spatial properties on the spectral characteristics of the attosecond pulses (25), we examine the variation of the on-axis spectrum at different positions (labelled (1) to (4)), for the generation position indicated by the dashed line. This is equivalent to examining the properties of

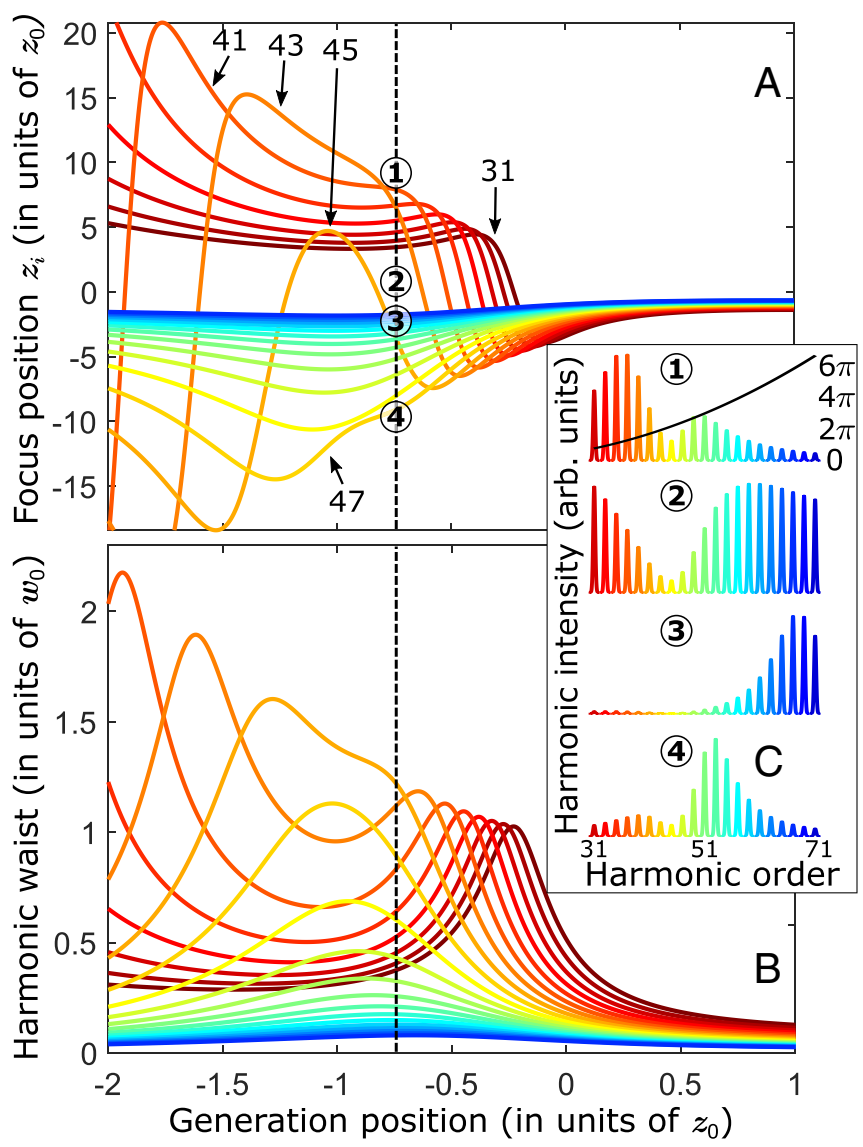

Fig. 7. Position of harmonic focus $z_{i}(A)$ and waist $(B)$ as a function of generation position for harmonics 31-71. The different harmonic orders are indicated by different rainbow color codes, from brown (31) to dark blue (71). $C$ shows harmonic spectra at four different positions along $z_{i}$, indicated from top to bottom by the numbered circles, for the generation position marked by the dashed line in $A$. The spectral phase of the attosecond pulse is shown in black for the first observation point, being largely independent of the observation position. arb. units, arbitrary units.
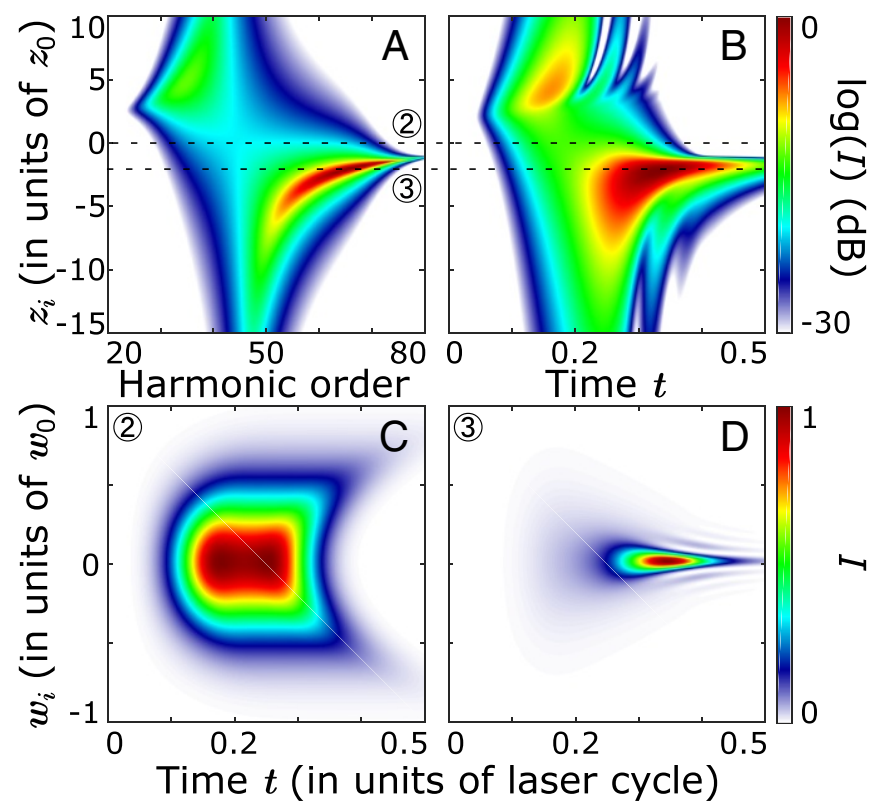

Fig. 8. ( $A$ and $B)$ Graphs show the on-axis spectral and temporal intensity, respectively, in logarithmic scales, as a function of the observation position, when generating at $z=-0.75 z_{0}$. The positions (2) and (3) (see Fig. $7 A$ ) are indicated by dashed lines. $C$ and $D$ show retrieved attosecond pulses at two different detection positions, i.e., (2) and (3) in Fig. 7A, in a linear scale.

the generated radiation after refocusing as illustrated in Fig. 1, for different "detection positions" in the focal region. We here assume equal strength of the generated harmonics, but account for the frequency variation in beam waist size (Fig. $7 B$ ) and position (Fig. $7 A$ ). The harmonic spectra shown in Fig. $7 C$ are found to be strongly dependent on the detection position, with, in some cases, strong bandwidth reduction and displacement of the central frequency. In contrast, the on-axis harmonic phase is dominated by the attosecond chirp, given by the last term in Eq. 6 and indicated by the black line in Fig. $7 C$, and does not vary much with the detection position.

\section{Spatiotemporal Coupling of Attosecond Pulses}

Finally, we estimate the influence of the chromatic aberrations on the temporal properties of the attosecond pulses. We consider a flat spectrum between harmonics 31 and 71 at the generation position indicated by the dashed line in Fig. 7, in the same conditions as in the previous paragraph. We propagate the harmonic fields using diffraction integrals and coherently add them to obtain the resulting attosecond pulse train in space and time at different detection positions. We take into account the different focus positions and divergences of the frequency components of the attosecond pulses, as well as the attosecond positive chirp.

Fig. 8 shows the spectral $(A)$ and temporal $(B)$ intensity (in color) of the generated attosecond pulse on-axis as function of the detection position relative to the generation position, here equal to $z=-0.75 z_{0}$ (dashed line in Fig. 7). In these conditions, the central frequency and pulse duration of the attosecond pulse vary distinctively, indicating strong spatiotemporal couplings. In particular, the high-frequency components (high harmonic orders) form a tight virtual focus before the generation position, while the low-frequency components have a more loose and real focus behind (Fig. 8A). The highest intensity is obtained before the generation position, while the shortest pulse is obtained afterward, as follows from Fig. $8 B$. The attosecond pulse is not the shortest at the generation position, where the spectral bandwidth is the largest, because the 
attosecond chirp stretches the pulse in time. Fig. $8 A$ and $B$ strikingly show that the shortest pulse and the highest intensity of the attosecond pulse are obtained in different positions, illustrating the difficulty of refocusing high-order harmonics, particularly for applications requiring high intensity.

Finally, Fig. $8 C$ and $D$ shows the spatiotemporal intensity profiles of the attosecond pulse at the positions where is it spectrally broadest $(C$, (2) ) and where it is most intense $(D$, (3)). The difference between the two cases is a signature of the strong spatiotemporal couplings of the generated attosecond pulses. These couplings, here studied at $z=-0.75 z_{0}$ (dashed line in Fig. 7), strongly depend on the position of generation.

\section{Conclusion}

In this work, we examine the focusing properties of high-order harmonics generated in gases. We use a simple Gaussian optics model, valid for a thin generating medium, assuming a fundamental Gaussian beam, and based on an analytical expression of the frequency- and intensity-dependent dipole phase. This model allows us to predict the focus and divergence of the two trajectory contributions to HHG. We validate the predictions of the model by numerical calculations based on solving the TDSE for the single-atom response and propagation equations for the response from the macroscopic medium. Experimental diver-

1. Akturk S, Gu X, Bowlan P, Trebino R (2010) Spatio-temporal couplings in ultrashort laser pulses. J Opt 12:093001-093020.

2. Miranda M, et al. (2014) Spatiotemporal characterization of ultrashort laser pulses using spatially resolved Fourier transform spectrometry. Opt Lett 39:5142-5145.

3. Pariente G, Gallet V, Borot A, Gobert O, Quéré $F$ (2016) Space-time characterization of ultra-intense femtosecond laser beams. Nat Photon 10:547-553.

4. Harth A, et al. (2017) Compact $200 \mathrm{kHz}$ HHG source driven by a few-cycle OPCPA. J Opt 20:014007.

5. Vincenti H, Quéré F (2012) Attosecond lighthouses: How to use spatiotemporally coupled light fields to generate isolated attosecond pulses. Phys Rev Lett 108:113904.

6. Kim KT, et al. (2013) Photonic streaking of attosecond pulse trains. Nat Photon 7 : 651-656.

7. Louisy M, et al. (2015) Gating attosecond pulses in a noncollinear geometry. Optica 2:563-566.

8. Sansone G, et al. (2006) Isolated single-cycle attosecond pulses. Science 314:443-446

9. Goulielmakis E, et al. (2008) Single-cycle nonlinear optics. Science 320:1614-1617.

10. Li J, et al. (2017) 53-attosecond X-ray pulses reach the carbon K-edge. Nat Commun 8:186.

11. Gaumnitz T, et al. (2017) Streaking of 43-attosecond soft-X-ray pulses generated by a passively CEP-stable mid-infrared driver. Opt Express 25:27506-27518.

12. Popmintchev T, et al. (2012) Bright coherent ultrahigh harmonics in the keV X-ray regime from mid-infrared femtosecond lasers. Science 336:1287-1291.

13. Cousin SL, et al. (2017) Attosecond streaking in the water window: A new regime of attosecond pulse characterization. Phys Rev X 7:041030.

14. Schafer KJ, Yang B, DiMauro LF, Kulander KC (1993) Above threshold ionization beyond the high harmonic cutoff. Phys Rev Lett 70:1599-1602.

15. Corkum PB (1993) Plasma perspective on strong-field multiphoton ionization. Phys Rev Lett 71:1994-1997.

16. Lewenstein M, Kulander KC, Schafer KJ, Bucksbaum PH (1995) Rings in abovethreshold ionization: A quasiclassical analysis. Phys Rev A 51:1495-1507.

17. Bellini M, et al. (1998) Temporal coherence of ultrashort high-order harmonic pulses. Phys Rev Lett 81:297-300.

18. Frumker E, Paulus GG, Niikura H, Villeneuve DM, Corkum PB (2009) Frequencyresolved high-harmonic wavefront characterization. Opt Lett 34:3026-3028.

19. Lloyd DT, O'Keeffe K, Anderson PN, Hooker SM (2016) Gaussian-Schell analysis of the transverse spatial properties of high-harmonic beams. Sci Rep 6:30504.

20. Johnson AS, et al. (2018) High-flux soft X-ray harmonic generation from ionizationshaped few-cycle laser pulses. Sci Adv 4:eaar3761.

21. Austin DR, et al. (2011) Lateral shearing interferometry of high-harmonic wavefronts. Opt Lett 36:1746.

22. Lee DG, Park JJ, Sung JH, Nam CH (2003) Wave-front phase measurements of high-order harmonic beams by use of point-diffraction interferometry. Opt Lett 28: 480-482.

23. Valentin C, et al. (2008) High-order harmonic wave fronts generated with controlled astigmatic infrared laser. J Opt Soc Am B 25:B161-B166.

24. Freisem L, Jansen GSM, Rudolf D, Eikema KSE, Witte S (2018) Spectrally resolved single-shot wavefront sensing of broadband high-harmonic sources. Opt Express 26:6860-6871.

25. Frumker E, et al. (2012) Order-dependent structure of high harmonic wavefronts. Opt Express 20:13870-13877.

26. Salières P, L'Huillier A, Lewenstein M (1995) Coherence control of high-order harmonics. Phys Rev Lett 74:3776-3779. gence measurements performed at the intense XUV beamline of the Lund Laser Centre show similar trends as those predicted by the numerical calculations, as well as by an extension of the Gaussian model, which includes the effect of a circular aperture. We also discuss the consequences of the fact that the harmonics have different focus positions and beam waists on the resulting spectra and pulse durations. The relative harmonic amplitudes are found to vary with the detection position, thus strongly affecting the spatiotemporal properties of the corresponding attosecond pulses.

The effects investigated in the present work have a strong impact on applications of attosecond pulses, requiring a small focal spot (e.g., to reach a high XUV intensity) over a broad bandwidth or during a short (attosecond) duration. These spatiotemporal couplings may be reduced by locating the generation medium after the laser focus and/or by minimizing the influence of the dipole phase, using a shaped fundamental beam $(53,54)$ or generating in waveguides (capillaries) $(47,55)$.

ACKNOWLEDGMENTS. This research was supported by the Swedish Research Council; the Swedish Foundation for Strategic Research; European Research Council Grant 339253 PALP; the Knut and Alice Wallenberg Foundation; and National Science Foundation Grant PHY-1713761. This project received funding from the European Union's Horizon 2020 research and innovation program under Marie Skłodowska-Curie Grant Agreements 641789 MEDEA and 793604 ATTOPIE.

27. Balcou $P$, Salières $P$, L'Huillier $A$, Lewenstein $M$ (1997) Generalized phase-matching conditions for high harmonics: The role of field-gradient forces. Phys Rev A 55:32043210 .

28. Quintard $L$ (2017) Caractérisation et contrôle des profils spatiaux spectraux et temporels de faisceaux XUV obtenus par génération d'harmoniques d'ordres élevés dans des gaz. PhD thesis (Université de Bordeaux, Bordeaux, France). French.

29. Quintard L, et al. (2018) Mirrorless focusing of XUV high-order harmonics. arxiv:1810.07282. Preprint, posted October 16, 2018.

30. Miyazaki K, Takada H (1995) High-order harmonic generation in the tunneling regime. Phys Rev A 52:3007-3021.

31. Tamaki Y, Itatini J, Nagata Y, Obara M, Midorikawa K (1999) Highly efficient phase matched high-harmonic generation by a self-guided laser beam. Phys Rev Lett 82:1422-1425.

32. Lai CJ, Kärtner FX (2011) The influence of plasma defocusing in high harmonic generation. Opt Express 19:22377-22387.

33. Bor Z (1989) Distortion of femtosecond laser pulses in lenses. Opt Lett 14:119-121.

34. Bor Z, Horváth ZL (1992) Distortion of femtosecond pulses in lenses. Wave optical description. Opt Commun 94:249-258.

35. Guo C, et al. (2018) Phase control of attosecond pulses in a train. J Phys B At Mol Opt Phys 51:034006.

36. Hernández-García C, San Román J, Plaja L, Picón A (2015) Quantum-path signatures in attosecond helical beams driven by optical vortices. New J Phys 17:093029.

37. Catoire F, et al. (2016) Complex structure of spatially resolved high-order-harmonic spectra. Phys Rev A 94:063401.

38. L'Huillier A, Balcou P, Candel S, Schafer KJ, Kulander KC (1992) Calculations of high order harmonic-generation processes in xenon at $1064 \mathrm{~nm}$. Phys Rev A 46:27782790 .

39. Lewenstein M, Balcou P, Ivanov M, L'Huillier A, Corkum P (1994) Theory of highorder harmonic generation by low-frequency laser fields. Phys Rev A 49:21172132.

40. Varjú K, et al. (2005) Frequency chirp of harmonic and attosecond pulses. J Mod Opt 52:379-394.

41. Carlström S, et al. (2016) Spatially and spectrally resolved quantum path interference with chirped driving pulses. New J Phys 18:123032.

42. Zaïr A, et al. (2008) Quantum path interferences in high-order harmonic generation Phys Rev Lett 100:143902.

43. Guo C (2018) A high repetition rate attosecond light source based on optical parametric amplification. Ph.D. thesis (Lund University, Lund, Sweden).

44. Rego L, San Román J, Picón A, Plaja L, Hernández-García C (2016) Nonperturbative twist in the generation of extreme-ultraviolet vortex beams. Phys Rev Lett 117:163202.

45. Saleh BEA, Teich MC (2007) Fundamentals of Photonics (John Wiley and Sons, New York).

46. Pérez-Hernández JA, Roso L, Plaja L (2009) Harmonic generation beyond the strong field approximation: The physics behind the short-wave-infrared scaling laws. Opt Express 17:9891-9903.

47. Durfee CG III, et al. (1999) Phase matching of high-order harmonics in hollow waveguides. Phys Rev Lett 83:2187-2190.

48. Manschwetus B, et al. (2016) Two-photon double ionization of neon using an intense attosecond pulse train. Phys Rev A 93:061402.

49. Coudert-Alteirac $\mathrm{H}$, et al. (2017) Micro-focusing of broadband high-order harmonic radiation by a double toroidal mirror. App/ Sci 7:1159. 
50. Horváth ZL, Bor Z (2003) Focusing of truncated Gaussian beams. Opt Commun 222: 51-68.

51. Gillen GD, Seck CM, Guha S (2010) Analytical beam propagation model for clipped focused-Gaussian beams using vector diffraction theory. Opt Express 18:4023-4040.

52. Macklin JJ, Kmetec JD, Gordon CL III (1993) High-order harmonic generation using intense femtosecond pulses. Phys Rev Lett 70:766-769.
53. Boutu W, et al. (2011) High-order-harmonic generation in gas with a flat-top laser beam. Phys Rev A 84:063406.

54. Dubrouil A, et al. (2011) Controlling high harmonics generation by spatial shaping of high-energy femtosecond beam. Opt Lett 36:2486-2488.

55. Popmintchev T, Chen MC, Arpin P, Murnane MM, Kapteyn HC (2010) The attosecond nonlinear optics of bright coherent X-ray generation. Nat Photon 4:822-832. 